\title{
ARTICLE
}

\section{Monte Carlo calculations for the determination of the radiation field in an interim storage facility}

\author{
Frank Becker $^{\mathrm{a}^{*}}$ and Guoqing Zhang ${ }^{\mathrm{a}, \mathrm{b}, \mathrm{c}}$ \\ ${ }^{a}$ Karlsruhe Institute of Technology, KIT, P.O. Box 3640, Karlsruhe, Germany; ${ }^{b}$ China Institute for Radiation Protection, P.O. Box \\ 120, Taiyuan, China; ' Shanghai Institute of Applied Physics, Chinese Academy of Science, Shanghai 201800, China
}

\begin{abstract}
Since currently there are no long term permanent storage places available, casks for storage and transport of radioactive material (CASTORs) are used to store spent nuclear fuel in interim storage facilities in Germany. For radiation protection purpose, simulations for CASTORs of type V/19 with MCNP5 were performed. The distribution of radiation sources in the containers and the complex composition of the thick walled CASTOR present a challenge for reliable simulations. In order to handle such complex simulations, variance reduction techniques were applied to improve the performance of the simulations. In particular, the weight window method was combined with the ability of MCNP5 to record a surface source. In this way the simulation time could be reduced to an acceptable level. Employing parallel computing we simulated CLUSTER arrangements with up to 64 CASTOR casks. Taking into account that the inhomogeneous field is difficult to measure, simulations yield new insights into dose rate distributions and notably into the composition of the mixed radiation field. An example for simulating the response of dosimeters in such fields demonstrates that simulations provide a powerful tool for radiation protection improvements.
\end{abstract}

Keywords: interim storage facilities; Monte Carlo; dose distribution; variance reduction; dosimeter response

\section{Introduction}

Mixed neutron-gamma fields still present a challenge for dosimetry. The interaction of neutrons with matter is more complex than for photons, which is reflected by the conversion coefficients from neutron fluence to ambient dose equivalent. A strong variation of about a factor 50 over an energy range from $10^{-1}$ to $10^{7} \mathrm{eV}$ is given [1].

Hence neutron dosimeters are not capable to measure the dose with the same accuracy as for photons. The strong dependence on the spectral distributions of the neutrons requires different calibration factors, which should be determined as accurate as possible.

An interim storage facility has been chosen as a representative example to investigate the behavior of an albedo dosimeter. The Monte Carlo code MCNP5 was employed to estimate the flux distributions of neutrons and gammas of the field. However, the simulation of the neutron-gamma field stemming from accumulated casks for storage and transport of radioactive materials (CASTORs) turned out to be time-consuming, if MCNP5 is used in the basic analog transport mode. To allow simulating geometries with 64 CASTOR-casks in acceptable times, variance reduction techniques and parallel computing has been applied. Moreover, surface source has been used to avoid unnecessary duplicated calculations.

\section{Materials and methods}

\subsection{MCNP5}

MCNP5 is a general-purpose Monte Carlo N-Particle code that can be used for neutron, photon, electron, or coupled neutron, photon, and electron transport. MCNP5 includes a general source, criticality source, and surface source, a rich collection of variance reduction techniques, as well as a flexible tally structure, and an extensive collection of cross-section data. The version MCNP5 1.51 [2] was employed.

\subsection{Model of CASTOR V/19}

The simplified model consisted of a single moderator layer and a uniform source distribution inside (Figure 1). The data for the simplified CASTOR V/19 model including the source information of spent fuels inside was provided by the Gesellschaft für Anlagen- und Reaktorsicherheit (GRS Garching) [3].

*Corresponding author. Email: frank.becker@kit.edu 


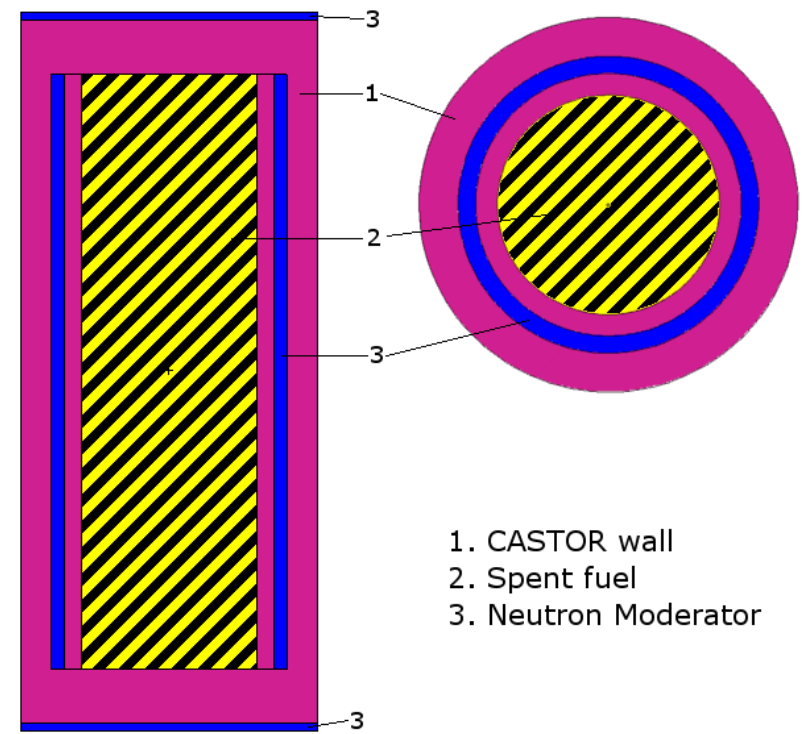

Figure 1. Model of CASTOR V/19.

\subsection{Weight windows and surface source}

Figure 2 shows the effect of mesh-based weight windows. Simulations of radiation sources in the thick walled CASTOR present a challenge for reliable results. For the investigated geometry, calculations without weight windows show particles barely penetrating the wall of the CASTOR. When weight windows are applied, for the same number of simulated source events, penetrating particles are now clearly visible. MCNP5 offers the possibility to generate surface sources. This option allows saving calculation time by avoiding the recalculation of the particle transport inside a CASTOR.
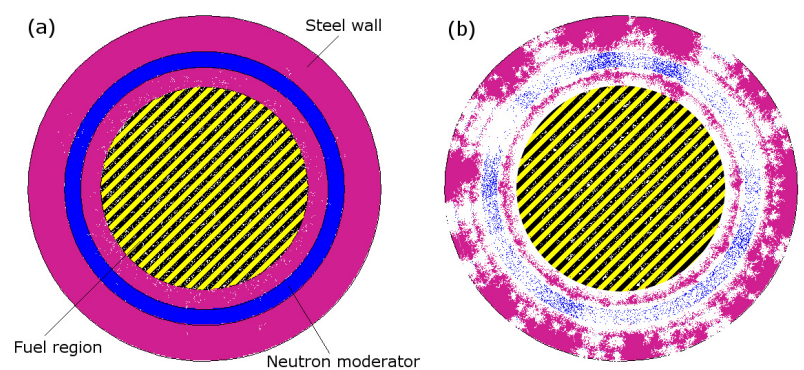

Figure 2. Cross section of the CASTOR showing particle distributions (white spots) obtained with different calculation methods. (a) Analog simulation, (b) weight windows.

In the present study the information of all particles which cross the outer surface of a CASTOR were stored into a file. This file can be used as a surface source in new simulations where the number of CASTORS of same type and content can be increased by placing multiple copies of the surface sources. This tool again helps to save computation time.

In Figure 3 examples for calculated spectra at the outer surface of a CASTOR are shown. Surface source simulations perform the random sampling of particles (including energy, position, weight and direction) based on the information stored in the surface source file, which is not an exact duplicate of analog simulations.
Hence the spectra look a little bit different. Taking into account the statistics, surface source simulations at the outside of a CASTOR could be considered as being equivalent to analog ones.
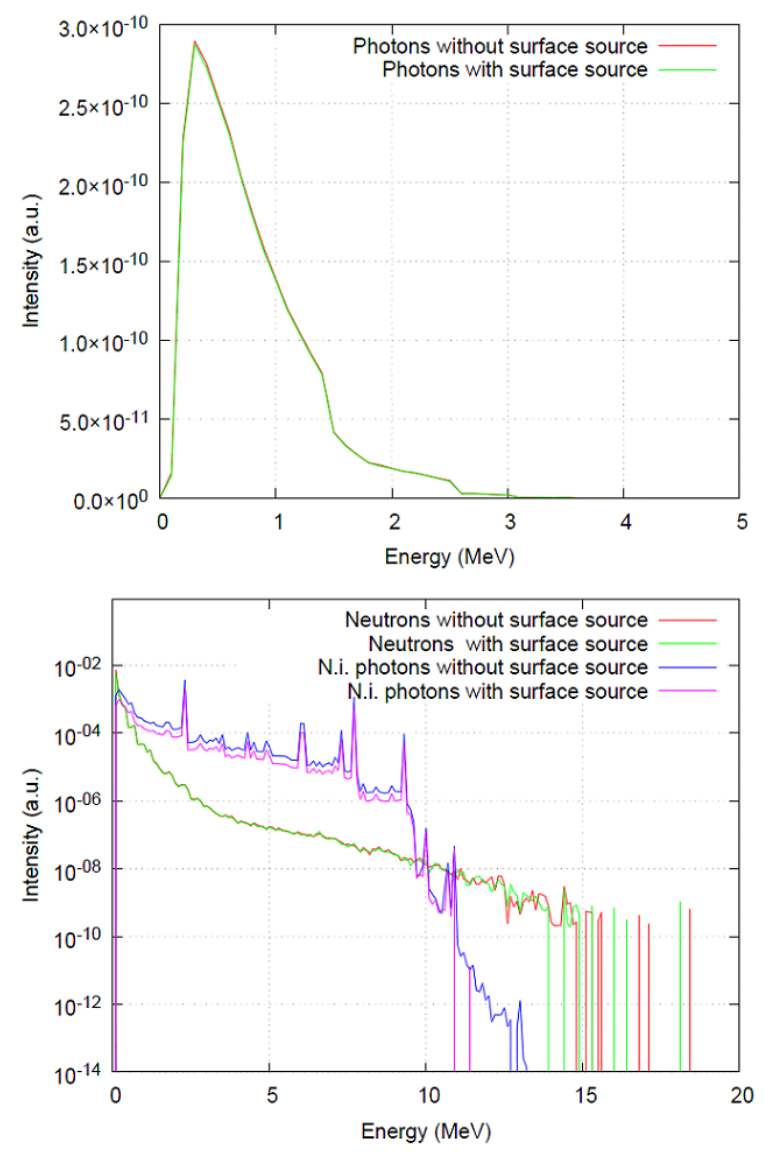

Figure 3. Calculated spectra of neutrons, photons and neutron induced (N.i.) photons at the outer surface of a CASTOR [6].

\subsection{Albedo dosimeter}

Albedo neutron dosimeters estimate the dose equivalent in the body by measuring the direct incident neutrons as well as so-called albedo neutrons. Various types of albedo dosimeters have been designed with different structures and materials [4]. Due to the complexity of neutron fields at different work places, calibration factors are employed to scale the dosimeter reading to a reference radiation field (e.g. Cf-252). However, to obtain a correct factor, the knowledge of the radiation field at the work place is needed. This can be achieved by measurements and/or simulations. Measurements, for example with Bonner spheres [5], can be very time-consuming and are in some cases difficult or not possible. In these cases simulations are an important tool to determine details of the radiation field and in turn the calibration factor. In this work an Alnor albedo dosimeter has been modeled and simulated with MCNP5 in the mixed neutron gamma field of an interim storage facility [6]. The relative response of the dosimeter to a Cf-252 reference field was also calculated to get the calibration factor. 


\section{Results and discussion}

\subsection{Weight windows, surface source and parallel computing}

An example for saving computation time when employing weight window and surface source options in comparison with the analog case is given in Table 1. The figure of merit, FOM, is given as the reciprocal value of the product of the squared relative calculated error and calculation time.

Table 1. Performance of the different methods (M): analog (A), weight window (W) and surface source (S).

\begin{tabular}{lccccc}
\hline $\begin{array}{l}\text { Source } \\
\text { particle } \\
\text { (fuel }\end{array}$ & $\begin{array}{c}\text { Tally } \\
\text { particle }\end{array}$ & M & $\begin{array}{c}\text { Compu- } \\
\text { tation } \\
\text { time } \\
\text { region) }\end{array}$ & $\begin{array}{c}\text { Relative } \\
\text { error }\end{array}$ & FOM \\
\hline Neutron & Neutron & A & $7.60 \times 10^{3}$ & $7 \times 10^{-4}$ & $2.69 \times 10^{2}$ \\
& & W & $5.63 \times 10^{3}$ & $4 \times 10^{-4}$ & $1.11 \times 10^{3}$ \\
& & S & $2.69 \times 10^{2}$ & $5 \times 10^{-4}$ & $1.44 \times 10^{5}$ \\
& Gamma & A & $7.60 \times 10^{3}$ & $1.2 \times 10^{-3}$ & $9.14 \times 10^{1}$ \\
& (neutron & W & $5.63 \times 10^{3}$ & $4 \times 10^{-4}$ & $1.11 \times 10^{3}$ \\
& induced) & S & $2.79 \times 10^{1}$ & $5 \times 10^{-4}$ & $1.44 \times 10^{5}$ \\
Gamma & Gamma & A & $4.33 \times 10^{4}$ & $8.67 \times 10^{-2}$ & $3.08 \times 10^{-3}$ \\
& & W & $5.36 \times 10^{3}$ & $4.0 \times 10^{-3}$ & $1.17 \times 10^{1}$ \\
& & S & $3.72 \times 10^{2}$ & $4.0 \times 10^{-3}$ & $1.68 \times 10^{3}$ \\
\hline
\end{tabular}

For the shown case with a single representative CASTOR V/19 weight windows can improve the performance (FOM) by about a factor of 5 for neutrons and about a factor of 10 for neutron-induced gammas. In particular for gamma source particles, weight windows improve by more than 2 orders of magnitude. Once a surface source is generated even more calculation time can be saved, since the transport calculations of source particles inside the CASTOR are omitted. Parallel computing with surface sources was also employed. In general the use of more processors is supposed to decrease the time of a calculation. However, for our scenario the situation occurred that the time of reading the surface source data could take longer than the time of the calculation on each processor. Depending on the number of CASTORs and processors involved, the use of more processors did not always result in a reduction of time for the simulation (for further information see [6]).

\subsection{Radiation field in an interim storage facility}

Figure 4 shows the calculated neutron dose rate distribution in an interim storage facility. The simulation model is based on data from the Philippsburg interim storage facility in Germany [6]. 64 CASTORs of the same type $V / 19$ and content are arranged inside the concrete storage building. The surface source method was applied. In the given scenario the calculated neutron dose is almost an order of magnitude higher than the gamma part so that only the neutron results are presented here. The maximum dose rates are close to 0.2 $\mathrm{mSv} / \mathrm{h}$. Note that the surface source option does not reflect the dose rates inside the CASTORs correctly.

Due to confidentiality reasons the content of the
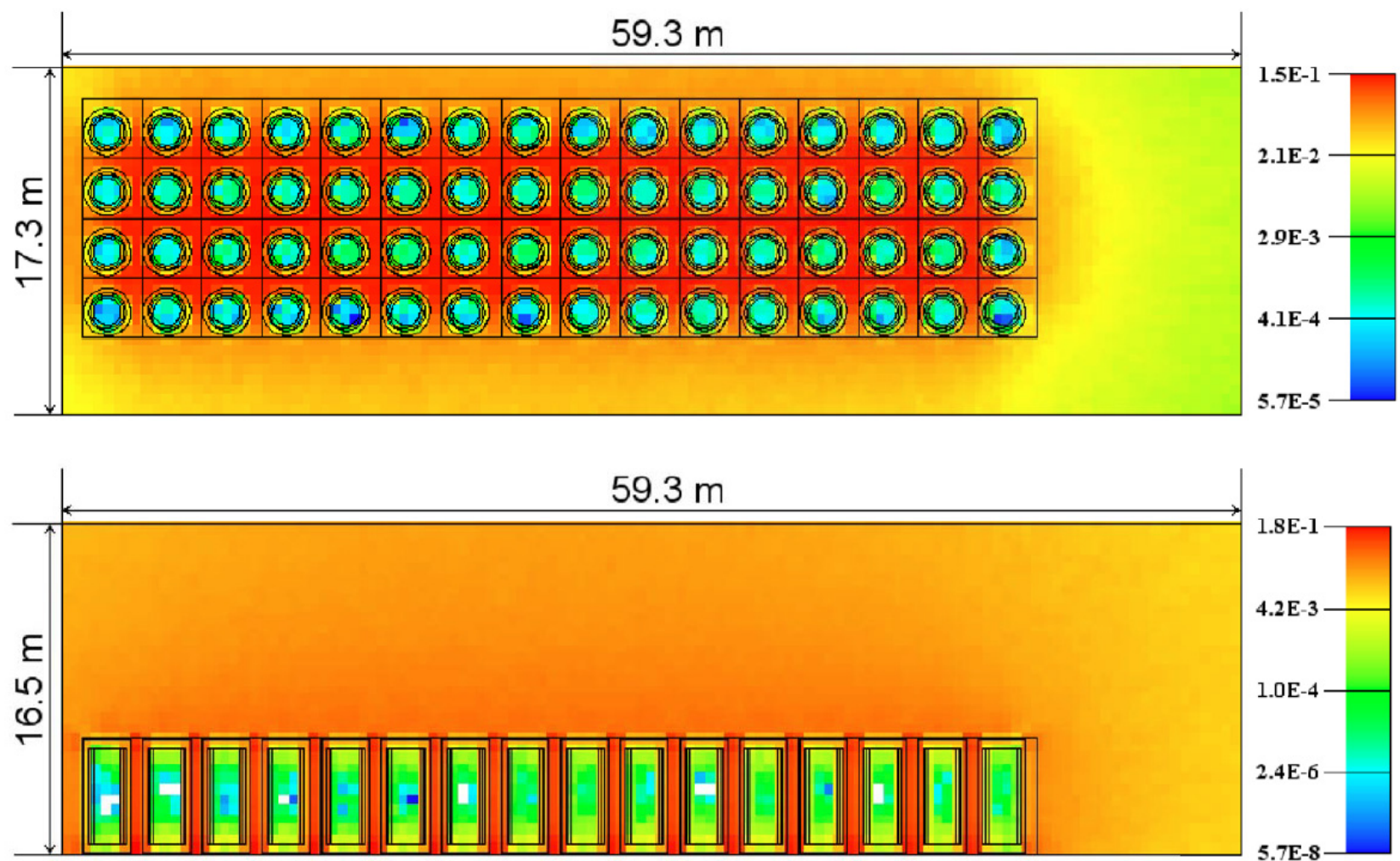

Figure 4. Dose rate distribution in units of $\mathrm{mSv} / \mathrm{h}$ for neutrons as simulated in a model of an interim storage building loaded with 64 CASTORs [6]. 
CASTORs are classified, so that a direct comparison of measured and simulated data was not feasible. However, doses measured (LB 6411, Berthold Technologies) in direct proximity to the CASTORS at the Philippsburg facility show neutron rates around 100 $\mu \mathrm{Sv} / \mathrm{h}$, which is in good agreement with our simulations.

The knowledge of the radiation field includes the spectra at different positions in the storage place. In this way it is possible to simulate the corresponding energy dependent response of a dosimeter.

\subsection{Albedo dosimeter}

Albedo dosimeters employ two detectors, which are sensitive to neutron (reading $\mathrm{M}_{\mathrm{n}}$ ) and gamma (reading $\mathrm{M}_{\gamma}$ ) radiation ( $\mathrm{Li}-6$ ), as well as two, which are sensitive to gamma radiation ( $\mathrm{Li}-7)$. One of each type is positioned behind a boron-loaded plastic encapsulation facing the backscatter body to measure at the albedo neutron position, the others face the radiation field to register field neutrons. The corresponding positions are labeled with $i$ and a in the lower right inset of Figure 5, respectively. The reading ratio $M_{n}(a) / M_{n}(i)$, which is related to the neutron field, can be used to find the albedo response $R_{n}(i)$ to estimate the neutron dose equivalent. This dose can be related to the standard calibration reading $\mathrm{R}_{\mathrm{nr}}(\mathrm{i})$ obtained with a Cf- 252 source. The gamma radiation response $\mathrm{R}_{\gamma}(\mathrm{i})$ is defined analog. According to the work of Burgkhardt and Piesch [7] an appropriate plotting of reading ratios allows to classify neutron fields into four categories N1 to N4.

N1 stands for reactors and accelerators, heavy shield-

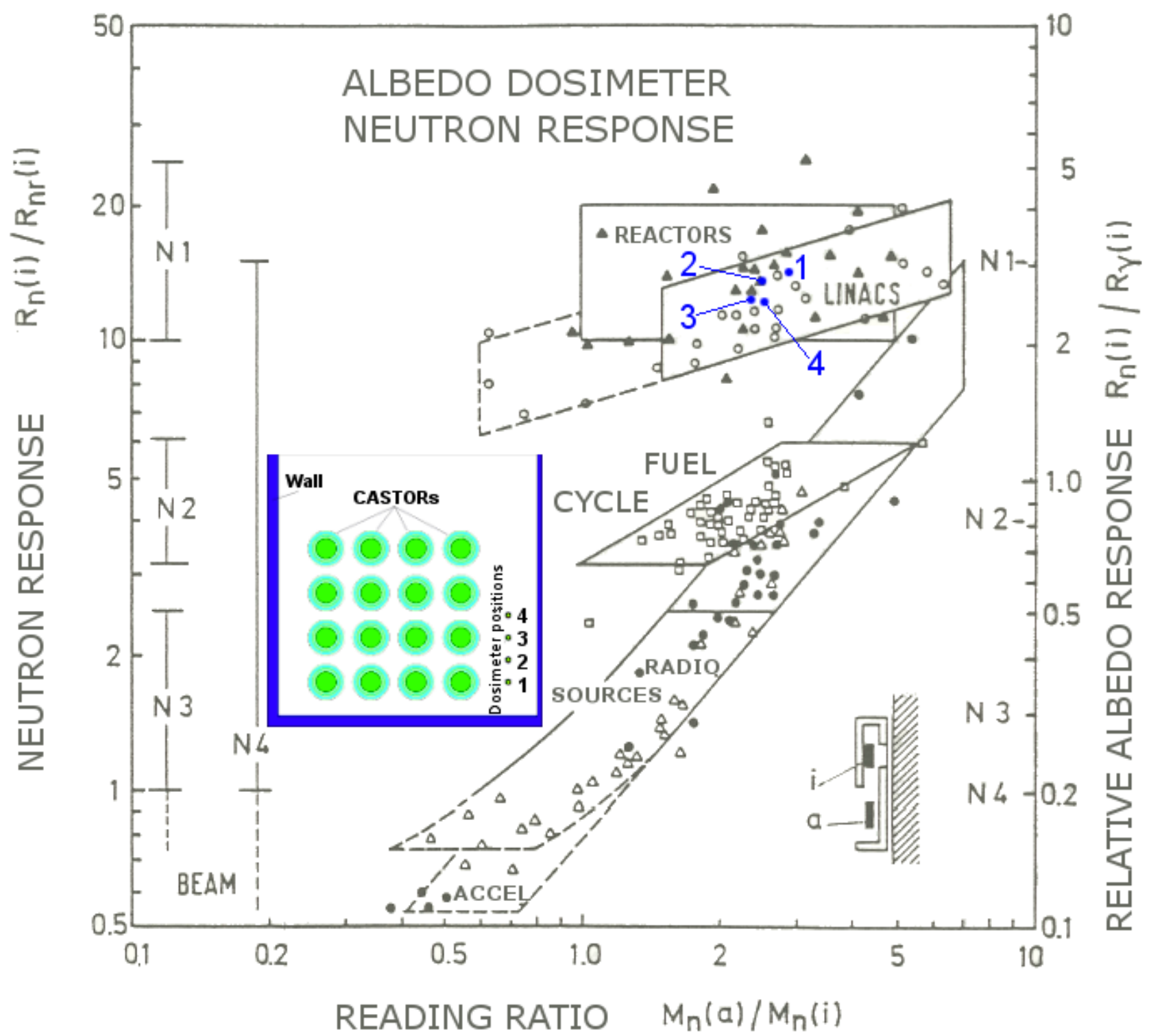

Figure 5. Neutron response of albedo dosimeter against the reading ratio of field neutrons $M_{n}(a)$ to albedo neutrons $M_{n}(i)$. $R_{n r}(i)$ is the response of detector in the reference field while $\mathrm{R}_{\gamma}(\mathrm{i})$ is the response to a reference gamma source (Cs-137). Four calculated results from this work are shown with blue dots/numbers. The inset shows the corresponding modeled setup with dosimeter positions 1 to 4 as well as 16 CASTORs in green surrounded by concrete walls marked in blue. Measured results are taken from reference [8]. 
ing, N2 for fuel element cycle, criticality, low shielding, $\mathrm{N} 3$ for radio nuclide neutron sources, and N4 for accelerators for research.

MCNP5 simulations with a model of an Alnor albedo dosimeter were performed at four different positions in an arrangement of 16 CASTORs as shown in the middle inset of Figure 5. The four positions were chosen to investigate the possible scattering and shielding influence of the walls and CASTORs. The number of $(n$, $\alpha$ ) reactions, which is proportional to the reading of the dosimeters, was used in the simulation to get the reading ratio $M_{n}(a) / M_{n}(i)$. The dose rates were obtained using the calculated fluence multiplied with dose conversion factors [9]. The results of the four positions are slightly different, which reflects the variation of the radiation field. However, the investigated Alnor dosimeter fits into category $\mathrm{N} 1$. In this scenario the dosimeter can be used in reactor areas as well as the interim storage place with the same calibration. For practical reasons this is an advantage when working in interim storage places close to reactor facilities. Hence, workers can employ the same dosimeter in both areas, so that a dosimeter changing procedure and the corresponding costs are avoided.

\section{Conclusion}

In the present work we show that Monte Carlo simulations are a helpful tool to investigate the complex radiation field for CASTORs and interim storage places. Moreover, simulations of dosimeters yield the information about their suitability and their calibration needs. In order to improve the performance of the complex simulations, variance reduction and surface source as well as parallel computation techniques are recommended. Simulations are an important tool to reveal details for radiation protection improvements, in particular when experiments are difficult. Key measurements to validate the simulations reduce the experimental effort to a minimum if reasonable experiments are feasible.

\section{Acknowledgements}

The authors would like to thank K. Hummelsheim and U. Hesse of GRS Garching for providing the source information of spent fuels inside the Castor V/19.

G. Zhang gratefully acknowledges the financial support of the Helmholtz-CSC Scholarship for his thesis at KIT.

\section{References}

[1] ICRU, Determination of operational dose equivalent quantities for neutrons, ICRU Report No. 66, Journal of the ICRU, 1(3) (2001).

[2] LANL, MCNP - A General Monte Carlo $N$-Particle Transport Code - Version 5, (2011), http://monp-green.lanl.gov/ (March 2011).

[3] K. Hummelsheim and U. Hesse, GRS Garching, Germany, private communication, 2009.

[4] E. Piesch and B. Burgkhardt, Albedo neutron dosimetry, Radiation Protection Dosimetry 10(1-4) (1985). pp. 175-188.

[5] R. L. Bramblett, R. I. Ewing and T. W. Bonner, New type of neutron spectrometer, Nuclear Instruments and Methods 9 (1960), pp. 1-12.

[6] G. Zhang, Monte Carlo Simulation of Mixed Neutron-Gamma Radiation Fields and Dosimetry Devices, Doctoral thesis, Institut für Biomedizinische Technik (IBT), KIT Karlsruhe, (2012), urn:nbn:de:swb:90-256840.

[7] E. Piesch and B. Burgkhardt, Field calibration technique for albedo neutron dosimeters, Radiation Protection Dosimetry 23(1/4) (1988), pp. 121-126.

[8] E. Piesch and B. Burgkhardt, Erprobung eines Albedoneutronendosimetriesystem: TLD Kalibrierund Meßverfahren, Neutronenkalibrierung, dosimetrische Eigenschaften, Routineanwendung, Tech. Rep. KfK 4303, Hauptabteilung Sicherheit, (1988).

[9] ICRP, Conversion Coefficients for use in Radiological Protection against External Radiation, ICRP Publication 74 (1997). 